\title{
A note on D-optimal chemical balance weighing designs with autocorrelated observations
}

\author{
Lukasz Smaga ${ }^{1}$
}

Received: 21 July 2014 / Revised: 3 March 2015 / Published online: 14 March 2015

(C) The Author(s) 2015. This article is published with open access at Springerlink.com

\begin{abstract}
In this paper, D-optimal chemical balance weighing designs with three objects are considered. The error terms are assumed to form a first-order autoregressive process, which implies that the covariance matrix of the vector of errors depends on the known parameter $\rho$. It is shown that the designs constructed by Katulska and Smaga (Metrika 76:393-407, 2013) are still D-optimal weighing designs with three objects under a wider interval of possible values for parameter $\rho$ than that considered in that paper. Those designs are also proved to be highly D-efficient designs, when D-optimal design is not known.
\end{abstract}

Keywords Autoregressive observations - Chemical balance weighing design . D-efficiency · D-optimality

Mathematics Subject Classification 62K05 - 05B20

\section{Introduction}

We will denote by $\mathcal{M}_{n \times p}( \pm 1)$ the set of all $n \times p$ matrices whose elements are all equal to -1 or 1 . Let us introduce a model of chemical balance weighing design with three objects. We use $n$ measuring operations from a chemical balance to estimate the true unknown weights $w_{1}, w_{2}, w_{3}$ of three objects. Assume that $y_{1}, \ldots, y_{n}$ are the observations in those $n$ operations respectively. Let observations follow the linear model $\mathbf{y}=\mathbf{X w}+\mathbf{e}$, where $\mathbf{y}=\left[y_{1}, \ldots, y_{n}\right]^{\prime}$ is an $n \times 1$ vector of observations,

Łukasz Smaga

1s@amu.edu.pl; lukaszsmaga17@gmail.com

1 Faculty of Mathematics and Computer Science, Adam Mickiewicz University, ul. Umultowska 87, 61-614 Poznan, Poland 
$\mathbf{X}=\left(x_{i j}\right) \in \mathcal{M}_{n \times 3}( \pm 1)$ is so-called design matrix, $\mathbf{w}=\left[w_{1}, w_{2}, w_{3}\right]^{\prime}$ is the vector of true unknown weights of objects, and $\mathbf{e}=\left[e_{1}, \ldots, e_{n}\right]^{\prime}$ is the vector of error components which form a first order autoregressive process. Therefore $\mathrm{E}(\mathbf{e})=\mathbf{0}_{n}$ is the $n \times 1$ null vector, and $\operatorname{Cov}(\mathbf{e})=1 /\left(1-\rho^{2}\right) \mathbf{S}$, where

$$
\mathbf{S}=\left[\begin{array}{cccccc}
1 & \rho & \rho^{2} & \cdots & \rho^{n-2} & \rho^{n-1} \\
\rho & 1 & \rho & \cdots & \rho^{n-3} & \rho^{n-2} \\
\rho^{2} & \rho & 1 & \cdots & \rho^{n-4} & \rho^{n-3} \\
\vdots & \vdots & \vdots & \ddots & \vdots & \vdots \\
\rho^{n-2} & \rho^{n-3} & \rho^{n-4} & \cdots & 1 & \rho \\
\rho^{n-1} & \rho^{n-2} & \rho^{n-3} & \cdots & \rho & 1
\end{array}\right]
$$

and $-1<\rho<1$. The matrix $\mathbf{S}$ is positive definite for $\rho \in(-1,1)$. In a chemical balance weighing design, if the $j$ th object is placed on the left (right) pan during the $i$ th weighing operation, then $x_{i j}=-1\left(x_{i j}=1\right)$. If the matrix $\mathbf{X}$ is of full column rank, then the generalized least-squares estimator of the vector $\mathbf{w}$ is equal to $\hat{\mathbf{w}}=\left(\mathbf{X}^{\prime} \mathbf{S}^{-1} \mathbf{X}\right)^{-1} \mathbf{X}^{\prime} \mathbf{S}^{-1} \mathbf{y}$, and its covariance matrix equals $1 /\left(1-\rho^{2}\right)\left(\mathbf{X}^{\prime} \mathbf{S}^{-1} \mathbf{X}\right)^{-1}$. The matrix $\mathbf{X}^{\prime} \mathbf{S}^{-1} \mathbf{X}$ is the information matrix of the design $\mathbf{X}$. In this paper, we only consider nonsingular designs, i.e. the designs with nonsingular information matrix.

An example of experiment (chemical process) described by the aforementioned model can be found in Jenkins and Chanmugam (1962). There are also papers in the literature relating to designs with other forms of $\operatorname{Cov}(\mathbf{e})$ (see, for example, Ceranka et al. 2006; Graczyk 2009; Masaro and Wong 2008) and spring balance weighing designs with $(0,1)$-design matrices (see, for instance, Graczyk 2012; Lopez and Neubauer 2010). The applications of weighing designs (in chemistry, medicine, economics, operations research etc.) are given in the following papers: Angelis et al. (2001); Banerjee (1975); Beckman (1973); Cheng (2014) and Graczyk (2013).

Our purpose is to find the best design with respect to D-optimality criterion. The design $\hat{\mathbf{X}}$ is D-optimal in the class of designs with three objects $\mathcal{M}_{n \times 3}( \pm 1)$ if $\operatorname{det}\left(\hat{\mathbf{X}}^{\prime} \mathbf{S}^{-1} \hat{\mathbf{X}}\right)=\max \left\{\operatorname{det}\left(\mathbf{X}^{\prime} \mathbf{S}^{-1} \mathbf{X}\right): \mathbf{X} \in \mathcal{M}_{n \times 3}( \pm 1)\right\}$, i.e. it maximizes the determinant of the information matrix among all designs with three objects. The inverse of $\mathbf{S}$ is equal to $1 /\left(1-\rho^{2}\right) \mathbf{A}$, where $\rho \in(-1,1)$ and $\mathbf{A}=\mathbf{I}_{n}+\rho^{2} \mathbf{K}_{1}-\rho \mathbf{K}_{2}, \mathbf{I}_{n}$ is the identity matrix of size $n, \mathbf{K}_{1}=\operatorname{diag}\left(0, \mathbf{1}_{n-2}^{\prime}, 0\right)$ and $\mathbf{K}_{2}$ is a tridiagonal $n \times n$ matrix with 0 on the diagonal, 1 on the first superdiagonal and on the first subdiagonal. Since $\mathbf{S}^{-1}=1 /\left(1-\rho^{2}\right) \mathbf{A}$, the design $\hat{\mathbf{X}}$ is D-optimal in the class $\mathcal{M}_{n \times 3}( \pm 1)$ if $\operatorname{det}\left(\hat{\mathbf{X}}^{\prime} \mathbf{A} \hat{\mathbf{X}}\right)=\max \left\{\operatorname{det}\left(\mathbf{X}^{\prime} \mathbf{A X}\right): \mathbf{X} \in \mathcal{M}_{n \times 3}( \pm 1)\right\}$.

When $\rho=0$, a lot of work has been done on developing optimal weighing design theory (see, for instance, Banerjee 1975; Cheng 2014; Jacroux et al. 1983; Neubauer 1997). In the case $\rho \neq 0$, it is harder to deal with optimality problems. Some results concerning optimal designs in this case are given for example in Angelis et al. (2001); Katulska and Smaga (2012, 2013); Li and Yang (2005); Smaga (2014) and Yeh and Lo Huang (2005), but many problems are still unsolved. In this paper, we extend the results of Katulska and Smaga (2013), where D-optimality of certain designs was proven under $\rho \in[0,1 /(n-2)]$. 
The remainder of this paper is organized as follows. In Sect. 2, we show that the D-optimal designs for $\rho \in[0,1 /(n-2)]$ constructed by Katulska and Smaga (2013) are still D-optimal under $\rho$ belonging to wider interval than $[0,1 /(n-2)]$. In Sect. 3, we give the lower bound for D-efficiency of weighing designs and we use it to show that the mentioned designs are highly D-efficient in cases, which are not included in Sect. 2. Some preliminary technical results are outlined in the Appendix.

We follow the notation of Yeh and Lo Huang (2005). For each vector $\mathbf{x}=$ $\left[x_{1}, \ldots, x_{n}\right]^{\prime} \in \mathcal{M}_{n \times 1}( \pm 1)$, we define the following numbers: cons $(\mathbf{x})=\#\left\{i: x_{i}=\right.$ $\left.x_{i+1}, 1 \leqslant i \leqslant n-1\right\}$, fcons $(\mathbf{x})=\min \left\{i: x_{i}=x_{i+1}, 1 \leqslant i \leqslant n-1\right\}$. For example, for $\mathbf{x}=[1,-1,1,1,-1,-1]^{\prime}$ we have cons $(\mathbf{x})=2$ and fcons $(\mathbf{x})=3$.

\section{D-optimal designs}

In this section, we extend the following theorem.

Theorem 1 (Katulska and Smaga 2013) Let $n \equiv 0(\bmod 4), \operatorname{Cov}(\mathbf{e})=1 /\left(1-\rho^{2}\right) \mathbf{S}$, where $\mathbf{S}$ is given by (1) and $\rho \in[0,1 /(n-2)]$. Then the design

$$
\tilde{\mathbf{X}}^{\prime}=\left\{\begin{array}{c}
{\left[\begin{array}{c}
<n>^{+} \\
<n / 2>^{+}<n / 2>^{-} \\
<n / 4>^{+}<n / 2>^{+}<n / 4>^{-}
\end{array}\right] \quad \text { if } \frac{n}{4}=2 k-1} \\
{\left[\begin{array}{c}
<n>^{+} \\
<n / 2>^{+}<n / 2>^{-} \\
<n / 4>^{+}<n / 2>^{-}<n / 4>^{+}
\end{array}\right] \quad \text { if } \frac{n}{4}=2 k,}
\end{array}\right.
$$

where

$$
\begin{aligned}
& <t>^{+}=\left[(-1)^{2},(-1)^{3},(-1)^{4}, \ldots,(-1)^{t+1}\right] \\
& <t>^{-}=\left[(-1)^{1},(-1)^{2},(-1)^{3}, \ldots,(-1)^{t}\right]
\end{aligned}
$$

and $k=1,2, \ldots$, is D-optimal design in the class $\mathcal{M}_{n \times 3}( \pm 1)$.

Unfortunately, it can be easily observed that $1 /(n-2) \rightarrow 0$ as $n \rightarrow \infty$. However, Theorem 1 in Katulska and Smaga (2013) implies the design $\widetilde{\mathbf{X}}$ given by (2) is Doptimal for all $\rho \in[0,1)$ in large subclass $\mathcal{M}_{n \times 3}( \pm 1) \backslash \mathcal{C}$, where $\mathcal{C}=\{\mathbf{X}=[\mathbf{x}, \mathbf{y}, \mathbf{z}]$ : $\operatorname{cons}(\mathbf{x})=0, \operatorname{cons}(\mathbf{y})=1, \operatorname{cons}(\mathbf{z})=1\}$. Furthermore, simulation studies suggest the design $\widetilde{\mathbf{X}}$ is D-better than any design in $\mathcal{C}$ for all $\rho \in[0,1)$. But, it is not easy to show this. We solve partially this problem in the following theorem.

Theorem 2 Assume that $n \equiv 0(\bmod 4), \operatorname{Cov}(\mathbf{e})=1 /\left(1-\rho^{2}\right) \mathbf{S}$, where $\mathbf{S}$ is given by (1), and $\rho \in[0,1)$ if $n=4,8, \ldots, 28$, and $\rho \in[0, \eta)$ if $n=32,36, \ldots$, where $\eta$ is the smallest positive root of the polynomial

$$
\begin{aligned}
p(\rho)= & \left(7 n^{2}-22 n-48\right) \rho^{5}-\left(n^{3}-41 n^{2}+124 n+240\right) \rho^{4} \\
& -\left(3 n^{3}-89 n^{2}+246 n+528\right) \rho^{3}-\left(3 n^{3}-87 n^{2}+192 n+592\right) \rho^{2}
\end{aligned}
$$




$$
-\left(n^{3}-40 n^{2}+56 n+320\right) \rho+8 n^{2}-64 .
$$

Then the design $\widetilde{\mathbf{X}}$ given by (2) is D-better than any design $\mathbf{X}=[\mathbf{x}, \mathbf{y}, \mathbf{z}] \in \mathcal{C}$.

Theorem 1 in Katulska and Smaga (2013) and Theorem 2 imply the following important corollary.

Corollary 1 Under the assumptions of Theorem 2, the design $\widetilde{\mathbf{X}}$ given by (2) is Doptimal in the class $\mathcal{M}_{n \times 3}( \pm 1)$.

For $n=4,8, \ldots, 28$, we significantly extend Theorem 1 for all $\rho \in[0,1)$ that is important, because we often do not need a large number of observations for three objects. When $n=32,36, \ldots$, Lemma 3 in the Appendix shows that the root $\eta$ of the polynomial $p$ given by (3) belongs to $(1 /(n-2), 1)$, and hence Theorem 1 is also improved for such $n$. The root $\eta$ can be derived numerically. For example, numerical approximation for this root for $n=32,36, \ldots, 60$ is $0.7599,0.4841,0.3754,0.3103,0.2657,0.2329,0.2076,0.1875$ respectively.

It is also worth mentioning that slight changes in the proofs of the necessary and sufficient conditions given in Katulska and Smaga (2013) under which the design with three objects is D-optimal actually show that those conditions still hold under the assumptions of Theorem 2. Unfortunately, it failed to prove D-optimality of the design $\widetilde{\mathbf{X}}$ given by (2) for all $\rho \in[0,1)$. However, in Sect. 3 we show that the design $\widetilde{\mathbf{X}}$ is highly D-efficient, when the assumptions of Theorem 2 do not hold.

In the following proof of Theorem 2, we use Budan-Fourier Theorem and an inequality given in Li (2013) which we recall in the Appendix.

Proof of Theorem 2 The main idea of the proof is to show that the inequality $\operatorname{det}\left(\widetilde{\mathbf{X}}^{\prime} \mathbf{A} \widetilde{\mathbf{X}}\right) \geqslant \operatorname{det}\left(\mathbf{X}^{\prime} \mathbf{A X}\right)$ holds for all designs $\mathbf{X}=[\mathbf{x}, \mathbf{y}, \mathbf{z}]$ such that $\operatorname{cons}(\mathbf{x})=0$, $\operatorname{cons}(\mathbf{y})=1$ and $\operatorname{cons}(\mathbf{z})=1$. By Lemma 3 in Katulska and Smaga (2013), we can suppose without loss of generality that $x_{1}=y_{1}=z_{1}=1$ and $\operatorname{fcons}(\mathbf{y})>$ fcons $(\mathbf{z})$. Lemmas 4 and 5 in Katulska and Smaga (2013) yield $\operatorname{det}\left(\widetilde{\mathbf{X}}^{\prime} \mathbf{A} \widetilde{\mathbf{X}}\right)=$ $\Delta(\Delta-4 \rho)(\Delta-8 \rho)-4 \rho^{2}(1+\rho)^{2}(\Delta-4 \rho)$ and $\mathbf{x}^{\prime} \mathbf{A x}=\Delta, \mathbf{y}^{\prime} \mathbf{A y}=\mathbf{z}^{\prime} \mathbf{A z}=\Delta-4 \rho$, $\mathbf{x}^{\prime} \mathbf{A y}=(2 \mathrm{fcons}(\mathbf{y})-n)(1+\rho)^{2}, \mathbf{x}^{\prime} \mathbf{A} \mathbf{z}=(2 \mathrm{fcons}(\mathbf{z})-n)(1+\rho)^{2}$ and $\mathbf{y}^{\prime} \mathbf{A z}=$ $(n-2$ fcons $(\mathbf{y})+2$ fcons $(\mathbf{z})-2)(1+\rho)^{2}+2(1+\rho)$, where $\Delta=(n-2)(1+\rho)^{2}+2(1+\rho)$. If $\rho=0$, then by Hadamard's inequality $\operatorname{det}\left(\widetilde{\mathbf{X}}^{\prime} \widetilde{\mathbf{X}}\right)=n^{3} \geqslant \operatorname{det}\left(\mathbf{X}^{\prime} \mathbf{X}\right)$. So assume that $\rho>0$. When $n=4$, there are three possible matrices exact to permuting columns:

$$
\mathbf{X}_{1}=\left[\begin{array}{rrr}
1 & 1 & 1 \\
-1 & 1 & -1 \\
1 & -1 & -1 \\
-1 & 1 & 1
\end{array}\right], \mathbf{X}_{2}=\left[\begin{array}{rrr}
1 & 1 & 1 \\
-1 & 1 & -1 \\
1 & -1 & 1 \\
-1 & 1 & 1
\end{array}\right], \mathbf{X}_{3}=\left[\begin{array}{rrr}
1 & 1 & 1 \\
-1 & -1 & -1 \\
1 & -1 & 1 \\
-1 & 1 & 1
\end{array}\right]
$$

Hence $\operatorname{det}\left(\mathbf{X}_{i}^{\prime} \mathbf{A} \mathbf{X}_{i}\right)=32(\rho+1)$ for $i=1,2,3$, and $\operatorname{det}\left(\widetilde{\mathbf{X}}^{\prime} \mathbf{A} \widetilde{\mathbf{X}}\right)=32 \rho^{3}+64 \rho^{2}+$ $96 \rho+64$. Thus $\operatorname{det}\left(\widetilde{\mathbf{X}}^{\prime} \mathbf{A} \widetilde{\mathbf{X}}\right)>\operatorname{det}\left(\mathbf{X}_{i}^{\prime} \mathbf{A} \mathbf{X}_{i}\right)$ for $i=1,2,3$. From now on we make the assumption: $n \geqslant 8$. We have divided the proof into two cases. 
Case 1 Let fcons $(\mathbf{y}) \neq n / 2$, fcons $(\mathbf{y}) \neq n / 2 \pm 1$, fcons $(\mathbf{z}) \neq n / 2$ and fcons $(\mathbf{z}) \neq$ $n / 2 \pm 1$. Lemma 2 in the Appendix implies the following inequality

$$
\begin{aligned}
\operatorname{det}\left(\mathbf{X}^{\prime} \mathbf{A X}\right) & \leqslant\left(\mathbf{x}^{\prime} \mathbf{A} \mathbf{x}\right)\left(\mathbf{y}^{\prime} \mathbf{A y}-\left(\mathbf{x}^{\prime} \mathbf{A} \mathbf{y}\right)^{2} /\left(\mathbf{x}^{\prime} \mathbf{A} \mathbf{x}\right)\right)\left(\mathbf{z}^{\prime} \mathbf{A z}-\left(\mathbf{x}^{\prime} \mathbf{A z}\right)^{2} /\left(\mathbf{x}^{\prime} \mathbf{A} \mathbf{x}\right)\right) \\
& =\Delta\left(\Delta-4 \rho-\left(\mathbf{x}^{\prime} \mathbf{A} \mathbf{y}\right)^{2} / \Delta\right)\left(\Delta-4 \rho-\left(\mathbf{x}^{\prime} \mathbf{A z}\right)^{2} / \Delta\right)
\end{aligned}
$$

By assumptions, $(2 \mathrm{fcons}(\mathbf{a})-n)^{2}(1+\rho)^{4} \geqslant 16(1+\rho)^{4}$ for $\mathbf{a} \in\{\mathbf{y}, \mathbf{z}\}$. Moreover $\Delta \leqslant n(1+\rho)^{2}$. By the above, the following inequality holds

$$
\operatorname{det}\left(\mathbf{X}^{\prime} \mathbf{A X}\right) \leqslant\left(\Delta(\Delta-4 \rho)-16(1+\rho)^{4}\right)\left(\Delta-4 \rho-16(1+\rho)^{4} /\left(n(1+\rho)^{2}\right)\right)
$$

Therefore

$$
\begin{aligned}
\operatorname{det}\left(\widetilde{\mathbf{X}}^{\prime} \mathbf{A} \widetilde{\mathbf{X}}\right)-\operatorname{det}\left(\mathbf{X}^{\prime} \mathbf{A} \mathbf{X}\right) \geqslant & \operatorname{det}\left(\widetilde{\mathbf{X}}^{\prime} \mathbf{A} \widetilde{\mathbf{X}}\right)-\left(\Delta(\Delta-4 \rho)-16(1+\rho)^{4}\right) \\
& \cdot\left(\Delta-4 \rho-16(1+\rho)^{2} / n\right) \\
= & 4(1+\rho) p(\rho) / n,
\end{aligned}
$$

where $p(\rho)$ is given by (3). So, to prove the inequality $\operatorname{det}\left(\widetilde{\mathbf{X}}^{\prime} \mathbf{A} \widetilde{\mathbf{X}}\right)>\operatorname{det}\left(\mathbf{X}^{\prime} \mathbf{A X}\right)$ we have to show that $p(\rho)>0$ for all $\rho$. It is easy to calculate that

$$
p(\rho)= \begin{cases}224 \rho^{5}+880 \rho^{4}+1664 \rho^{3}+1904 \rho^{2}+1280 \rho+448 & \text { if } n=8 \\ 696 \rho^{5}+2448 \rho^{4}+4152 \rho^{3}+4448 \rho^{2}+3040 \rho+1088 & \text { if } n=12 \\ 1392 \rho^{5}+4176 \rho^{4}+6032 \rho^{3}+6320 \rho^{2}+4928 \rho+1984 & \text { if } n=16 \\ 2312 \rho^{5}+5680 \rho^{4}+6152 \rho^{3}+6368 \rho^{2}+6560 \rho+3136 & \text { if } n=20 \\ 3456 \rho^{5}+6576 \rho^{4}+3360 \rho^{3}+3440 \rho^{2}+7552 \rho+4544 & \text { if } n=24 \\ 4824 \rho^{5}+6480 \rho^{4}+\rho\left(7520-3496 \rho^{2}\right)+\left(6208-3616 \rho^{2}\right) & \text { if } n=28 .\end{cases}
$$

Thus $p(\rho)>0$ for all $\rho \in(0,1)$ when $n=8,12, \ldots, 28$. For $n=32,36, \ldots$, by Lemma 3 in the Appendix the polynomial $p$ has exactly one root $\eta$ in the interval $(0,1)$, and hence $p(\rho)>0$ for all $\rho \in(0, \eta)$, because $p(0)=8 n^{2}-64>0$.

Case 2 Let fcons $(\mathbf{y})=n / 2$ or fcons $(\mathbf{y})=n / 2 \pm 1$ or $f \operatorname{cons}(\mathbf{z})=n / 2$ or fcons $(\mathbf{z})=$ $n / 2 \pm 1$. We give the proof only for the case fcons $(\mathbf{y})=n / 2$; the proofs for the other cases are similar. Let $a=$ fcons $(\mathbf{z})$. We have

$$
\begin{aligned}
\operatorname{det}\left(\mathbf{X}^{\prime} \mathbf{A X}\right)= & \Delta(\Delta-4 \rho)^{2}-(2 a-n)^{2}(1+\rho)^{4}(\Delta-4 \rho) \\
& -4(1+\rho)^{2}[(a-1)(1+\rho)+1]^{2} \Delta .
\end{aligned}
$$

Hence $\operatorname{det}\left(\widetilde{\mathbf{X}}^{\prime} \mathbf{A} \widetilde{\mathbf{X}}\right)-\operatorname{det}\left(\mathbf{X}^{\prime} \mathbf{A} \mathbf{X}\right)=(1+\rho) f(a)$, where

$$
\begin{aligned}
f(a)= & 8(1+\rho)^{3}\left[(n-2) \rho^{2}+2(n-2) \rho+n\right] a^{2} \\
& -4(1+\rho)^{3}\left[\left(n^{2}-4\right) \rho^{2}+2 n(n-2) \rho+n^{2}\right] a+n^{2}(n-2) \rho^{5}
\end{aligned}
$$




$$
\begin{aligned}
& +n\left(5 n^{2}-16 n+16\right) \rho^{4}+\left(10 n^{3}-36 n^{2}+48 n-32\right) \rho^{3} \\
& +n\left(10 n^{2}-32 n+32\right) \rho^{2}+n^{2}(5 n-10) \rho+n^{3} .
\end{aligned}
$$

We consider $f$ as a quadratic function of the variable $a$. The discriminant of $f$ is equal to $-16(1+\rho)^{3} w(\rho)$, where

$$
\begin{aligned}
w(\rho)= & \left(n^{4}-8 n^{3}+16 n^{2}-16\right) \rho^{7} \\
& +\left(7 n^{4}-60 n^{3}+152 n^{2}-96 n-48\right) \rho^{6} \\
& +\left(21 n^{4}-180 n^{3}+496 n^{2}-480 n+80\right) \rho^{5} \\
& +\left(35 n^{4}-280 n^{3}+736 n^{2}-736 n+240\right) \rho^{4} \\
& +n\left(35 n^{3}-240 n^{2}+512 n-352\right) \rho^{3} \\
& +n^{2}\left(21 n^{2}-108 n+136\right) \rho^{2}+n^{3}(7 n-20) \rho+n^{4} .
\end{aligned}
$$

Using Budan-Fourier Theorem (see Theorem 4 in the Appendix), we can show that all coefficients of the polynomial $w(\rho)$, considered as polynomials of variable $n$, do not have any roots in the interval $(7, \infty)$ (for more detailed argumentation of this kind we refer to the proof of Lemma 3 in the Appendix). Therefore all these coefficients are positive for $n \in(7, \infty)$. Hence the discriminant of $f$ is negative. So, $f(a)>0$ for all $a$, since the coefficient of $a^{2}$ in $f$ is positive. This finishes the proof.

\section{D-efficiency of design $\tilde{X}$}

In this section, we show that the design $\widetilde{\mathbf{X}}$ of the form (2) is highly D-efficient, when D-optimal designs in $\mathcal{M}_{n \times 3}( \pm 1)$ are not known.

Following the definition of Bulutoglu and Ryan (2009), the D-efficiency of a design $\mathbf{X} \in \mathcal{M}_{n \times p}( \pm 1)$ is defined by the formula

$$
\operatorname{D-eff}(\mathbf{X})=\left[\frac{\operatorname{det}\left(\mathbf{X}^{\prime} \mathbf{A} \mathbf{X}\right)}{\max _{\mathbf{Y} \in \mathcal{M}_{n \times p}( \pm 1)} \operatorname{det}\left(\mathbf{Y}^{\prime} \mathbf{A Y}\right)}\right]^{1 / p}
$$

under $\operatorname{Cov}(\mathbf{e})=1 /\left(1-\rho^{2}\right) \mathbf{S}$, where $\mathbf{S}$ is given by (1). Under the assumptions of Theorem 2, $\max _{\mathbf{Y} \in \mathcal{M}_{n \times 3}( \pm 1)} \operatorname{det}\left(\mathbf{Y}^{\prime} \mathbf{A Y}\right)=\operatorname{det}\left(\widetilde{\mathbf{X}}^{\prime} \mathbf{A} \widetilde{\mathbf{X}}\right)$ by Corollary 1, and hence D-eff $(\widetilde{\mathbf{X}})=1$. However, when the assumptions of Theorem 2 do not hold, value of the denominator of D-eff $(\mathbf{X})$ is unknown and we can not compute the D-efficiency of $\mathbf{X} \in \mathcal{M}_{n \times 3}( \pm 1)$. But, in the following lemma, we give the lower bound for it. Next we use this lower bound for the D-efficiency of weighing design to show that the design $\widetilde{\mathbf{X}}$ of the form (2) has high D-efficiency when $n \geqslant 32$ and $\rho \geqslant \eta$, where $\eta$ is defined in Theorem 2.

Lemma 1 If $n \equiv 0(\bmod 4), \operatorname{Cov}(\mathbf{e})=1 /\left(1-\rho^{2}\right) \mathbf{S}$, where $\mathbf{S}$ is given by $(1), \rho \in(0,1)$ and $\Delta=(n-2)(1+\rho)^{2}+2(1+\rho)$, then the D-efficiency of nonsingular design $\mathbf{X} \in \mathcal{M}_{n \times 3}( \pm 1)$ is greater than 


$$
\left[\frac{\operatorname{det}\left(\mathbf{X}^{\prime} \mathbf{A} \mathbf{X}\right)}{\Delta(\Delta-4 \rho)^{2}}\right]^{1 / 3} .
$$

Proof Let $\mathbf{Y}=\left[\mathbf{y}_{1}, \mathbf{y}_{2}, \mathbf{y}_{3}\right] \in \mathcal{M}_{n \times 3}( \pm 1)$ be an arbitrary nonsingular design. Hadamard's inequality implies the determinant of the matrix $\mathbf{Y}^{\prime} \mathbf{A Y}$ is less than or equal to the product of diagonal elements of this matrix, i.e.

$$
\operatorname{det}\left(\mathbf{Y}^{\prime} \mathbf{A Y}\right) \leqslant\left(\mathbf{y}_{1}^{\prime} \mathbf{A y}_{1}\right)\left(\mathbf{y}_{2}^{\prime} \mathbf{A} \mathbf{y}_{2}\right)\left(\mathbf{y}_{3}^{\prime} \mathbf{A} \mathbf{y}_{3}\right)
$$

By Lemma 4 in Katulska and Smaga (2013), we have $\mathbf{y}_{i}^{\prime} \mathbf{A} \mathbf{y}_{i}=\Delta-4 \xi \rho$ for $i=1,2,3$, where $\xi=\operatorname{cons}\left(\mathbf{y}_{i}\right)$. For $\mathbf{a} \in \mathcal{M}_{n \times 1}( \pm 1)$, it is easy to see that $\mathbf{a}^{\prime} \mathbf{A a}=\Delta$ if and only if $\mathbf{a}$ is the vector of ones or the vector of minus ones. Since $\mathbf{Y}$ is nonsingular, there exists at most one $i$ such that $\mathbf{y}_{i}^{\prime} \mathbf{A y}_{i}=\Delta$. Therefore $\operatorname{det}\left(\mathbf{Y}^{\prime} \mathbf{A Y}\right) \leqslant \Delta(\Delta-4 \rho)^{2}$, and hence

$$
\max _{\mathbf{Y} \in \mathcal{M}_{n \times 3}( \pm 1)} \operatorname{det}\left(\mathbf{Y}^{\prime} \mathbf{A Y}\right) \leqslant \Delta(\Delta-4 \rho)^{2}
$$

So, by (4),

$$
D-\operatorname{eff}(\mathbf{X}) \geqslant\left[\frac{\operatorname{det}\left(\mathbf{X}^{\prime} \mathbf{A} \mathbf{X}\right)}{\Delta(\Delta-4 \rho)^{2}}\right]^{1 / 3}
$$

We now show that the equality in (5) never holds. Suppose that the equality in (5) holds. Then $\operatorname{det}\left(\mathbf{Y}^{\prime} \mathbf{A Y}\right)=\Delta(\Delta-4 \rho)^{2}$ for some nonsingular $\mathbf{Y}=\left[\mathbf{y}_{1}, \mathbf{y}_{2}, \mathbf{y}_{3}\right]$ in the class $\mathcal{M}_{n \times 3}( \pm 1)$. By Hadamard's inequality, the last equality holds if and only if $\mathbf{Y}^{\prime} \mathbf{A Y}$ is diagonal matrix and its diagonal elements are equal to $\Delta, \Delta-4 \rho, \Delta-4 \rho$, in any order (see Theorem 7.8.1 in Horn and Johnson 2013, p.505). Without loss of generality, we can assume that $\mathbf{y}_{1}^{\prime} \mathbf{A} \mathbf{y}_{1}=\Delta$ and $\mathbf{y}_{2}^{\prime} \mathbf{A} \mathbf{y}_{2}=\mathbf{y}_{3}^{\prime} \mathbf{A} \mathbf{y}_{3}=\Delta-4 \rho$. By Lemma 4 in Katulska and Smaga (2013), $\operatorname{cons}\left(\mathbf{y}_{1}\right)=0$ and $\operatorname{cons}\left(\mathbf{y}_{i}\right)=1$ for $i=2$, 3. Hence the equalities $\mathbf{y}_{1}^{\prime} \mathbf{A} \mathbf{y}_{2}=0$ and $\mathbf{y}_{1}^{\prime} \mathbf{A} \mathbf{y}_{3}=0$ imply that $\operatorname{fcons}\left(\mathbf{y}_{i}\right)=n / 2$ for $i=2,3$ (see Lemma 5 (c) in Katulska and Smaga 2013). But, then the design $\mathbf{Y}$ is singular. Contradiction.

Theorem 3 Let $n \equiv 0(\bmod 4), n \geqslant 32, \operatorname{Cov}(\mathbf{e})=1 /\left(1-\rho^{2}\right) \mathbf{S}$, where $\mathbf{S}$ is given by (1) and $\rho \in[\eta, 1)$, where $\eta$ is defined in Theorem 2. Then with an increase in $\rho$, the lower bound for the D-efficiency of $\widetilde{\mathbf{X}}$ becomes smaller. Moreover, D-eff $(\widetilde{\mathbf{X}})$ is greater than 0.988 .

Proof From Lemma 1 and from the proof of Theorem 2, we conclude that the lower bound for the D-efficiency of $\widetilde{\mathbf{X}}$ is equal to

$$
\left[\frac{\Delta(\Delta-8 \rho)-4 \rho^{2}(1+\rho)^{2}}{\Delta(\Delta-4 \rho)}\right]^{1 / 3} .
$$


Since $\Delta=(n-2)(1+\rho)^{2}+2(1+\rho)$, it can be written as follows

$$
\left[\frac{((n-2)(1+\rho)+2)(\Delta-8 \rho)-4 \rho^{2}(1+\rho)}{((n-2)(1+\rho)+2)(\Delta-4 \rho)}\right]^{1 / 3} \text {. }
$$

Denote it by $f(\rho)$ and consider as a function of $\rho \in(0,1)$. Its derivative is equal to

$$
\frac{4 n(1+\rho) w(\rho)-64 \rho^{4}}{3(n \rho+n-2 \rho)^{2}\left(n(\rho+1)^{2}-2 \rho(\rho+3)\right)^{2}(f(\rho))^{2 / 3}},
$$

where $w(\rho)=\left(n^{2}-8 n+20\right) \rho^{3}+\left(n^{2}-6 n+4\right) \rho^{2}-n(n-2) \rho-n^{2}$. For the polynomial $w$, we have $M(0)=M(1)=1$, where $M(c)$ is defined in Theorem 4 in the Appendix. So, by Budan-Fourier Theorem (see Theorem 4 in the Appendix), the polynomial $w$ does not have any roots in $(0,1)$. Since the numbers $w(0)$ and $w(1)$ are negative, $w(\rho)<0$ for all $\rho \in(0,1)$. Therefore $\partial f(\rho) /(\partial \rho)<0$ for all $\rho \in(0,1)$, and hence $f(\rho)$ is decreasing. So, D-eff $(\widetilde{\mathbf{X}})$ is greater than the right hand side of (7) at $\rho=1$, i.e. D-eff $(\widetilde{\mathbf{X}})>\left(1-n /\left(n^{2}-3 n+2\right)\right)^{1 / 3}$. Let the function $g, g:(31, \infty) \rightarrow \mathbb{R}$ be defined by $g(x)=\left(1-x /\left(x^{2}-3 x+2\right)\right)^{1 / 3}$. We have $g^{\prime}(x)=\left(x^{2}-2\right) /\left(x^{2}-3 x+2\right)^{2}>0$ for all $x>31$, so $g$ is increasing. Thus D-eff $(\widetilde{\mathbf{X}})>g(32)=0.9883963$.

Theorem 3 implies the lower bound for the D-efficiency of $\widetilde{\mathbf{X}}$ given by (6) becomes smaller with an increase in $\rho$. However, the decrease of it is at most $1.2 \%$, because D-eff $(\widetilde{\mathbf{X}})>0.988$. So, the design $\widetilde{\mathbf{X}}$ is highly D-efficient design in the class $\mathcal{M}_{n \times 3}( \pm 1)$, when $n \geqslant 32$ and $\rho \geqslant \eta$, where $\eta$ is defined in Theorem 2 . This result is the next reason, which together with results of Sect. 2, confirms the conjecture that the $\operatorname{design} \widetilde{\mathbf{X}}$ is D-optimal in $\mathcal{M}_{n \times 3}( \pm 1)$, when $n \equiv 0(\bmod 4), \operatorname{Cov}(\mathbf{e})=1 /\left(1-\rho^{2}\right) \mathbf{S}$, where $\mathbf{S}$ is given by (1) and $\rho \in[0,1)$.

Open Access This article is distributed under the terms of the Creative Commons Attribution License which permits any use, distribution, and reproduction in any medium, provided the original author(s) and the source are credited.

\section{Appendix}

We first recall Budan-Fourier Theorem (see, for instance, Conkwright 1943), which we use in the proofs of Theorems 2 and 3 and in the proof of Lemma 3.

Theorem 4 (Budan-Fourier) Assume that $w$ is a polynomial of degree $n$ with real coefficients. Let $M(c)$ denote the number of variations of sign in the sequence $w(x), w^{\prime}(x), w^{\prime \prime}(x), \ldots, w^{(n)}(x)$, when $x=c$, where $c$ is any real number. Then the number of roots of the polynomial $w$ in the interval $(a, b), a<b$, is $M(a)-M(b)-2 k$, where $k$ is a positive integer or zero.

In the proof of Theorem 2, we also use the following lemma, which follows directly from Corollary 3.2 in $\mathrm{Li}$ (2013), since a positive definite matrix is a $K_{0}$-matrix. 
Lemma 2 (Li 2013) If $\mathbf{B}=\left[b_{i j}\right]$ is real symmetric positive definite matrix of size $n$, with $\prod_{i=1}^{n} b_{i i}>0$, then $\operatorname{det}(\mathbf{B}) \leqslant b_{11} \prod_{i=2}^{n}\left(b_{i i}-b_{1 i}^{2} / b_{11}\right)$.

We now consider the behavior of the root $\eta$ of the polynomial $p$ given by (3).

Lemma 3 Let $n=32,36, \ldots$. The polynomial $p$ given by (3) of variable $\rho$ has exactly one root in the interval $(0,1)$ and this root is greater than $1 /(n-2)$.

Proof It is easy to calculate that $p(0)=8 n^{2}-64, p^{\prime}(0)=-\left(n^{3}-40 n^{2}+56 n+320\right)$, $p^{(2)}(0)=-2\left(3 n^{3}-87 n^{2}+192 n+592\right), p^{(3)}(0)=-6\left(3 n^{3}-89 n^{2}+246 n+528\right)$, $p^{(4)}(0)=-24\left(n^{3}-41 n^{2}+124 n+240\right), p^{(5)}(0)=120\left(7 n^{2}-22 n-48\right)$. For $n=32,36$ the sequence of signs of the components of the above sequence equals ++--++ . Consider each component of the above sequence as a polynomial of variable $n$. It can be calculated that for each of these polynomials we have $M(39)-$ $M(\infty)=0$. By Budan-Fourier Theorem, each of these polynomials does not have any roots in $(39, \infty)$. Hence $p(0)>0, p^{(i)}(0)<0, i=1, \ldots, 4$, and $p^{(5)}(0)>0$ for $n=40,44, \ldots$ Thus when $n=40,44, \ldots$, the sequence of signs of the components of the above sequence is equal to +----+ . Therefore $M_{p}(0)=2$. In much the same way as above, we show that $M_{p}(1 /(n-2))=2$ and $M_{p}(1)=1$. Hence Budan-Fourier Theorem implies the polynomial $p$ has exactly one root in the interval $(1 /(n-2), 1)$, and it does not have any roots in $(0,1 /(n-2))$, which completes the proof.

\section{References}

Angelis L, Bora-Senta E, Moyssiadis C (2001) Optimal exact experimental designs with correlated errors through a simulated annealing algorithm. Comput Stat Data Anal 37:275-296

Banerjee KS (1975) Weighing designs for chemistry, medicine, economics, operations research, statistics. Marcel Dekker Inc., New York

Beckman RJ (1973) An application of multivariate weighing designs. Commun Stat 1:561-565

Bulutoglu DA, Ryan KJ (2009) D-optimal and near D-optimal $2^{k}$ fractional factorial designs of resolution V. J Stat Plan Inference 139:16-22

Ceranka B, Graczyk M, Katulska K (2006) A-optimal chemical balance weighing design with nonhomogeneity of variances of errors. Stat Probab Lett 76:653-665

Cheng CS (2014) Optimal biased weighing designs and two-level main-effect plans. J Stat Theory Pract 8:83-99

Conkwright NB (1943) An elementary proof of the Budan-Fourier theorem. Am Math Mon 50:603-605

Graczyk M (2009) Regular A-optimal design matrices $\mathbf{X}=\left(x_{i j}\right)$ with $x_{i j}=-1$, 0, 1. Stat Pap 50:789-795

Graczyk M (2012) A-optimal spring balance weighing designs under some conditions. Commun StatTheory Methods 41:2386-2393

Graczyk M (2013) Some applications of weighing designs. Biom Lett 50:15-26

Horn RA, Johnson CR (2013) Matrix analysis, 2nd edn. Cambridge University Press, Cambridge

Jacroux M, Wong CS, Masaro JC (1983) On the optimality of chemical balance weighing designs. J Stat Plan Inference 8:231-240

Jenkins GM, Chanmugam J (1962) The estimation of slope when the errors are autocorrelated. J R Stat Soc. Ser B (Stat Methodol) 24:199-214

Katulska K, Smaga $Ł$ (2012) D-optimal chemical balance weighing designs with $n \equiv 0(\bmod 4)$ and 3 objects. Commun Stat-Theory Methods 41:2445-2455

Katulska K, Smaga $Ł$ (2013) D-optimal chemical balance weighing designs with autoregressive errors. Metrika 76:393-407

Li CH, Yang SY (2005) On a conjecture in D-optimal designs with $n \equiv 0(\bmod 4)$. Linear Algebra Appl 400:279-290 
Li Y (2013) On improvements of Fischer's inequality and Hadamard's inequality for $K_{0}$-matrices. J Inequal Appl 2013:460

Lopez EA, Neubauer MG (2010) D-optimal (0,1)-weighing designs for 10 objects. Linear Multilinear Algebra 58:151-171

Masaro J, Wong CS (2008) D-Optimal designs for correlated random vectors. J Stat Plan Inference 138:4093-4106

Neubauer MG (1997) An inequality for positive definite matrices with applications to combinatorial matrices. Linear Algebra Appl 267:163-174

Smaga $€$ (2014) Necessary and sufficient conditions in the problem of D-optimal weighing designs with autocorrelated errors. Stat Probab Lett 92:12-16

Yeh HG, Lo Huang MN (2005) On exact D-optimal designs with 2 two-level factors and $n$ autocorrelated observations. Metrika 61:261-275 